\title{
Efetividade da simulação realística no ensino de cuidados paliativos em enfermagem: estudo randomizado
}

\section{Effectiveness of realistic simulation in teaching palliative care in nursing: a randomized study}

\section{Efectividad de la simulación realista en la enseñanza de cuidados paliativos en enfermería: un estudio aleatorizado}

\author{
Margareth Alves Bastos e Castro ${ }^{1}$, Wendell Costa Bazílio², Taís Alves de Morais ${ }^{3}$, \\ Jussara Regina Martins ${ }^{4}$, Rita de Cássia Almeida ${ }^{5}$
}

Informações do Artigo: Recebido em: 13/10/2020 Aceito em: 02/11/2020
DOI:

RESUMO

Objetivo: Avaliar a efetividade da estratégia da simulação realística no ensino de cuidados paliativos entre estudantes de enfermagem. Metodologia: Ensaio randomizado controlado. Participaram 23 estudantes de enfermagem; 12, no grupo controle, que realizou pré-teste, palestra e pós-teste; 11, na intervenção, grupo com pré-teste, palestra, simulação realística e pós-teste. Resultados: Os dados relacionados à média de respostas corretas no pré-teste com o pós-teste apresentaram significância estatística $(p=0,029)$. Na comparação entre as médias de sucessos com e sem simulação, apenas a não simulação foi significativa $(p=0,044)$ e para os participantes que realizaram a simulação não houve significância $(p=0,391)$. Conclusão: $A$ estratégia da simulação realística foi efetiva no ensino de cuidados paliativos entre os graduandos, evidenciado pelo escore positivo entre um primeiro e o segundo momento quando avaliados por meio de um teste cognitivo. DESCRITORES:

Enfermagem; Treinamento por simulação; Cuidados paliativos; Ensino.

${ }^{1}$ Enfermeira. Faculdade de Ciências Médicas e da Saúde de Juiz de Fora. E-mail: maghms@terra.com.br. Endereço: Faculdade de Ciências Médicas e da Saúde de Juiz de Fora, Curso de Enfermagem. Endereço: Alameda Salvaterra, $\mathrm{n}^{\circ}$ 200, Salvaterra, Juiz de Fora - MG, CEP: 36.033-003.

Enfermeiro. Faculdade de Ciências Médicas e da Saúde de Juiz de Fora. E-mail: wendell.bazilio@gmail.com

${ }^{3}$ Enfermeira. Hospital Santa Casa de Belo Horizonte. E-mail: tais2alves@gmail.com

4Enfermeira. Faculdade de Ciências Médicas e da Saúde de Juiz de Fora. E-mail: jussaramartinsjf@gmail.com

${ }^{5}$ Enfermeira. Faculdade de Ciências Médicas e da Saúde de Juiz de Fora. E-mail: ritaenfa@yahoo.com.br 
ABSTRACT

Objective: To evaluate the effectiveness of the realistic simulation strategy in teaching palliative care among nursing students. Methodology: Randomized controlled trial. 23 nursing students participated; 12, in the control group, which performed a pre-test, lecture and post-test; 11, in the intervention, group with pre-test, lecture, realistic simulation and post-test. Results: The data related to the mean of correct answers in the pre-test with the post-test presented statistical significance $(p=0.029)$. The comparison between the means of successes with and without simulation, only the nonsimulation was significant $(p=0.044)$ and for the participants who performed the simulation there was no significance $(p=0.391)$. Conclusion: The strategy of realistic simulation was effective in teaching palliative care among undergraduate students, evidenced by the positive score between the first and the second moments when evaluated using a cognitive test.

DESCRIPTORS:

Nursing; Simulation training; Palliative care; Teaching.

\section{RESUMEN}

Objetivo: Evaluar la efectividad de la estrategia de simulación realista en la enseñanza de cuidados paliativos en estudiantes de enfermería. Metodología: Ensayo controlado aleatorio. Participaron 23 estudiantes de enfermería; 12, en el grupo de control, que realizó una prueba previa, una conferencia y una prueba posterior; 11, en la intervención, grupo con pre-test, conferencia, simulación realista y post-test. Resultados: Los datos relacionados con el promedio de respuestas correctas en la prueba previa con la prueba posterior mostraron significación estadística $(p=0,029)$. Al comparar los promedios de éxitos con y sin simulación, solo la no simulación fue significativa $(p=0.044)$ y para los participantes que realizaron la simulación no hubo significación $(p=0.391)$. Conclusión: La estrategia de simulación realista fue efectiva en la enseñanza de cuidados paliativos entre estudiantes de pregrado, evidenciado por la puntuación positiva entre el primer y el segundo momento cuando se evaluó mediante una prueba cognitiva.

DESCRIPTORES:

Enfermería; Capacitación en simulación; Cuidados paliativos; Enseñando.

\section{INTRODUÇÃO}

Mudanças pedagógicas vêm acontecendo no sentido de romper com paradigmas tradicionais no ensino em enfermagem. Contribuindo com esse fato, e com a intenção de se proporcionar uma formação mais qualificada, as metodologias ativas, cujo foco central é o estudante, poderão corroborar a formação interdisciplinar crítica e reflexiva do enfermeiro. Neste sentido a simulação realística tem se destacado como relevante nesse processo(1-3).

Como ressaltado nas Diretrizes Curriculares Nacionais, a atuação do enfermeiro vai muito além de uma técnica realizada: assistir na enfermagem envolve conhecimento técnico-científico e deve considerar os aspectos biológicos, emocionais, culturais, sociais e espirituais do paciente, da família e da comunidade(3). É preciso saber atuar desde a prevenção de doenças até o cuidar direto dos pacientes cuja cura não é mais possível. Este último caso merece atenção, visto que é pouco abordado na maioria dos cursos de graduação em enfermagem(4,5).

Apesar do tema cuidado paliativo não ser adotado obrigatoriamente nos currículos de 
graduação em enfermagem, é inevitável para o professor não trabalhar com tal temática em sala de aula, pois é preciso que o enfermeiro seja capaz de atuar em situações de doenças com prognóstico limitante. Com o aumento da população idosa, observa-se, além do impacto financeiro ao sistema de saúde, a elevação no número de doenças crônicas não transmissíveis como o câncer, diabetes, doenças cardiovasculares, muitas delas diagnosticadas em estágio avançado e com nenhuma chance de recuperação(5,6).

A definição de cuidado paliativo pela Organização Mundial de Saúde (OMS), em 2002, considera a assistência, diante de problemas com a saúde, sob uma perspectiva de aprimoramento da qualidade de vida, tanto dos pacientes quanto de seus familiares, mediante a prevenção e alívio do sofrimento; precoce identificação, avaliação e tratamento da dor e outros problemas com olhar para aspectos físicos, psicossociais e espirituais( ${ }^{(7)}$.

Saber atuar no processo de finitude implica em aliviar o sofrimento do paciente e da família. Cabe ao enfermeiro, para atender esta demanda, trabalhar de forma organizada e sistematizada com métodos científicos e referenciais teóricos, sendo de significativa relevância a capacitação desse enfermeiro em cuidados paliativos(8).

$\mathrm{Na}$ atualidade o cuidado paliativo, apesar de bastante falado, é pouco praticado em todas as instituições de saúde, portanto, a realização dessa prática durante a graduação é difícil de ser vivenciada. Além disso, este obstáculo pode ser acompanhado do processo de ensino ainda meramente tradicional, cujo conteúdo é apresentado aos estudantes de enfermagem, por meio de aulas expositivas e pouco aprofundado, o que pode significar uma lacuna mediante a necessidade exigida na prática clínica do enfermeiro(9).

Pretende-se demonstrar que, com a utilização de novas estratégias de ensino, será possível favorecer a aquisição de conhecimentos teóricos entre os estudantes de enfermagem relacionados aos cuidados em fim de vida. Ademais, durante a graduação as disciplinas não deverão focar somente a doença, mas permitir que o aluno amplie seu entendimento em relação ao cuidar, inclusive quando não há possibilidade de cura(10).

Para o ensino de cuidados paliativos e assistência do enfermeiro, docentes devem refletir se metodologias ativas, como a simulação, favorecerão o ensino relativo a este tema, uma vez que ainda existe uma carência de estudos com essa abordagem no Brasil. Novas evidências precisam ser apresentadas dissertando essa temática e analisando-a sob a perspectiva do uso nas escolas brasileiras. Nesse contexto, questiona-se se o avanço nas metodologias pedagógicas de ensino poderá ser efetivo à formação do enfermeiro para atuar com pacientes cuja cura não seja mais possível. 


\section{Objetivo}

Avaliar a efetividade da estratégia da simulação realística no ensino de cuidados paliativos entre estudantes de enfermagem.

\section{METODOLOGIA}

\section{Desenho, local do estudo e período}

Ensaio clínico randomizado controlado. Esse estudo foi desenvolvido em sala de aula e no laboratório de enfermagem de um curso de enfermagem na Zona da Mata de Minas Gerais durante o primeiro semestre de 2017.

\section{População}

Participaram 23 estudantes do curso de enfermagem do quarto ao sétimo períodos divididos em dois grupos: controle, grupo que assistiu à palestra e não participou da sessão de simulação realística e intervenção, grupo que participou da palestra e da sessão de simulação realística. Os estudantes foram divididos aleatoriamente nos grupos acima conforme uma tabela de randomização gerada por site (www.random.org) em computador, a qual foi realizada previamente ao momento da coleta de dados da pesquisa.

\section{Critérios de inclusão e exclusão}

Foram considerados critérios de elegibilidade os estudantes maiores de 18 anos, que estavam regularmente matriculados no curso de graduação em enfermagem entre o quarto e sétimo períodos no primeiro semestre de 2017. Estar licenciado ou ausente na data de coleta dos dados foram os critérios adotados para exclusão.

\section{Protocolo do estudo}

Os estudantes dos quarto, quinto, sexto e sétimo períodos foram inicialmente selecionados por meio da lista de matrículas efetivadas até 15 de março de 2017. Posteriormente, foram solicitados a fazerem parte do estudo, fato que ocorreu no período compreendido entre os dias 16 a 25 de março de 2017, quando os acadêmicos, nos intervalos e ao final das aulas, foram instados a participarem da pesquisa por meio de convite pessoal dos pesquisadores, momento este em que foram esclarecidas a finalidade do estudo, a maneira como ele se organizaria e a data para a coleta de dados, em 01 de abril de 2017. Nesse momento, não receberam informação se seriam do grupo controle ou do grupo 
intervenção. A coleta de dados foi realizada pelos pesquisadores na data mencionada acima, sábado, durante o período da manhã.

Inicialmente, após a chegada dos 23 estudantes representando os quatro períodos participantes do estudo, eles foram alocados em uma sala de aula. A partir dessa ação o grupo estava orientado a não utilizar nenhum instrumento de comunicação, a não se comunicar entre si ou com qualquer outra pessoa até o encerramento da coleta de dados. Todos os participantes permaneceram sob a supervisão de dois pesquisadores durante a coleta de dados. Este Momento 1 foi composto pela realização de uma avaliação cognitiva (pré-teste) contendo 10 afirmativas as quais foram julgadas em concordo (C) e discordo (D) por eles com duração de 15 minutos.

Após recolhimento do pré-teste e mantida a ausência de comunicação entre os acadêmicos, na mesma sala, eles assistiram a uma palestra do tipo aula expositiva sobre o tema "A assistência de enfermagem no tratamento paliativo", com duração de 40 minutos e mais cinco minutos para perguntas. A palestra foi ministrada por uma enfermeira e professora do curso de enfermagem da instituição cenário e com conhecimento específico na área de cuidados paliativos.

Enquanto a palestra era realizada, foi aplicada a randomização (www.random.org) entre os estudantes presentes de cada período para definir em qual grupo cada um seria alocado - grupo controle (pré-teste; palestra; pós-teste) e grupo intervenção (pré-teste; palestra; simulação; pós-teste). 0 grupo controle teve 12 estudantes envolvidos e 11, o grupo intervenção.

Encerrada a palestra, os estudantes foram comunicados a qual grupo pertenciam. Os participantes do grupo controle permaneceram na sala e realizaram imediatamente o Momento 2 (pósteste) composto de um teste de avaliação cognitiva (pós-teste) com questões idênticas ao primeiro momento para avaliar a aquisição de conhecimento cognitivo relacionada à assistência de enfermagem no tratamento paliativo. Os estudantes participantes da intervenção foram encaminhados para 0 laboratório de enfermagem acompanhados da professora responsável pela sessão de simulação.

Para a sessão de simulação, os 11 estudantes novamente randomizados (www.random.org) tiveram as seguintes funções: três acadêmicos exerceram a função de enfermeiros e outros dois a de técnicos de enfermagem e os demais assistiram à simulação sem comunicação entre si. A sessão de simulação realística teve como objetivo primário de aprendizagem identificar a necessidade da instalação de medidas de conforto para um paciente em tratamento paliativo, portador de câncer para a melhora da qualidade de vida do mesmo e, como objetivos secundários de aprendizagem desenvolver a comunicação eficaz com o paciente, seus familiares e a equipe.

A sessão de simulação foi realizada com a participação de um ator profissional (que fez 0 paciente simulado portador de câncer em tratamento paliativo) e duas atrizes (agentes comunitárias de 
saúde que representaram a esposa e a filha do paciente simulado). Ambos estavam caracterizados e foram previamente treinados pelos pesquisadores. O cenário de simulação foi testado pelos pesquisadores previamente à sua execução.

Chegando ao laboratório de enfermagem, os estudantes já designados em suas funções, por meio da professora facilitadora, foram apresentados (briefing) ao cenário e a todos os recursos para 0 atendimento ao paciente. Foi descrito o caso clínico e o papel de cada participante. Em seguida, foi iniciada a sessão de simulação. O cenário teve a duração de sete minutos, sendo o tempo todo gravado.

Encerrada a sessão de simulação, imediatamente foi realizado o debriefing com duração de 22 minutos, almejando refletir sobre os aspectos do atendimento que deveriam ser mantidos e os que se modificariam, caso tivessem que atender o mesmo paciente. 0 debriefing foi realizado obedecendo a três fases: a descrição com duração de quatro minutos; 16 minutos para discussão e, dois minutos para a conclusão com a finalização da descrição dos objetivos de aprendizagem do caso proposto. Encerrado este momento, os estudantes imediatamente realizaram o teste de avaliação cognitiva (pós-teste) com questões idênticas ao primeiro momento para avaliar a aquisição do conhecimento cognitivo relacionada à assistência de enfermagem no tratamento paliativo idem ao já realizado pelo grupo controle.

$O$ instrumento de coleta de dados constou de um formulário elaborado por professores da área de enfermagem em oncologia e foi do tipo teste de avaliação cognitiva. Este teste continha 10 afirmativas sobre a assistência de enfermagem no tratamento paliativo, as quais foram julgadas pelos participantes em concordo $(C)$ e discordo (D) no pré-teste e no pós-teste. Inicialmente, no pré-teste, os estudantes foram questionados sobre idade, gênero, etnia e período em curso na graduação em enfermagem.

\section{Análise dos resultados e estatística}

Os dados do estudo foram tabulados por meio do Statistical Package for the Social Sciences (SPSS) versão 15 e a estatística descritiva foi utilizada para apresentação dos resultados das características demográficas: idade, gênero, etnia e períodos do curso. Foi realizada estatística inferencial em que os grupos de intervenção e controle foram comparados no baseline quanto aos escores presentes no somatório de acertos das afirmativas da avaliação cognitiva (pré-teste) no intuito de ver se existiam diferenças de conhecimento cognitivo entre os grupos. Posteriormente, os grupos (controle e intervenção) foram comparados entre si quanto à pontuação na realização do pós-teste (avaliação cognitiva). Para análise da normalidade dos dados foi aplicado o teste de Shapiro-Wilk e, em ambas as avaliações de escore, foi aplicado o teste $T$ pareado para medidas independentes $\mathrm{e}$ considerado $p<0,05$ como significante. 


\section{Aspectos éticos}

Este estudo obedeceu à Resolução $n^{0} 466 / 12$, do Conselho Nacional de Saúde/MS que esclarece sobre as diretrizes e normas regulamentadoras de pesquisas envolvendo seres humanos e preconizou a preservação dos participantes e da Instituição. A coleta de dados foi iniciada a partir da liberação do parecer $n^{0}$ 1. 877.872 do Comitê de Ética e Pesquisa com Seres Humanos. Todos os participantes foram esclarecidos pelos pesquisadores sobre o estudo bem como seus objetivos, em seguida leram o Termo de Consentimento Livre e Esclarecido e, após o aceite em participar da pesquisa, assinaram o mesmo. Este estudo está registrado no banco brasileiro de registro de ensaios clínicos sobre o número, RBR-6n3mw9.

\section{RESULTADOS}

Foram acompanhados 23 estudantes do quarto ao sétimo períodos do curso de enfermagem, sendo a maioria pertencente ao gênero feminino $(n=17)$. $O$ fluxograma dos participantes encontra-se demonstrado na figura 1.

\section{Figura 1. Fluxograma do ensaio clínico}

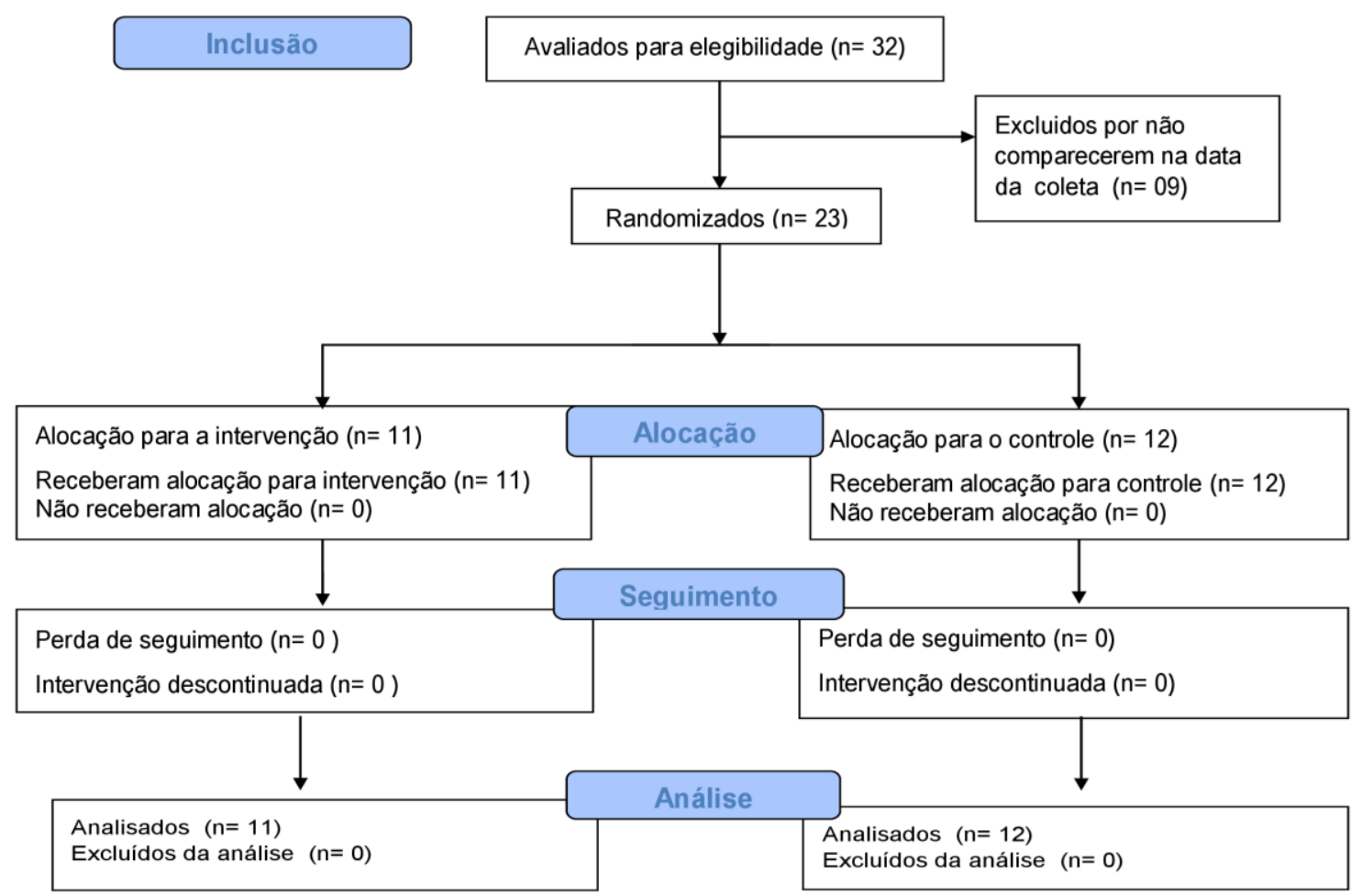

Fonte: Dados da pesquisa, 2017.

Considerando-se as características sociodemográficas dos participantes em ambos os grupos, foram observados que $17(73,9 \%)$ participantes eram do gênero feminino e seis (26.0\%) do gênero 
masculino; quanto a etnia $12(52,1 \%)$ se consideraram brancos, seis $(26,0 \%)$ negros e cinco $(21,7 \%)$ pardos. Relacionado ao período em curso da graduação em enfermagem a maioria dos participantes, sete $(30,4 \%)$ estavam no $5^{\circ}$ período, seis $(26,0 \%)$ no $5^{\circ}$ período, cinco $(21,7 \%)$ do $7^{\circ}$ e os outros cinco $(21,7 \%)$ no $4^{\circ}$ período.

A idade dos participantes variou de 19 a 44 anos, com média de 26,2 $\pm 6,7$ anos com intervalo de confiança $(I C)=[23,3 ; 29,1]$. Não houve diferença estatística $(p=0,270)$ nesses valores e entre os períodos que participaram ou não da simulação $(0,919)$.

Os dados relacionados à média de acertos no pré-teste com o pós-teste apresentaram significância estatística $(p=0,029)$. Já a comparação entre as médias de acertos com e sem simulação, somente a sem simulação apresentou significância estatística $(p=0,044)$ e para os participantes que realizaram a simulação não houve significância estatística $(p=0,391)$, conforme se verifica nas tabelas 1 e 2.

Tabela 1. Número de acertos no pré-teste e no pós-teste entre os grupos controle e intervenção

\begin{tabular}{cccc}
\hline $\begin{array}{c}\text { Número de acertos em } \\
10 \text { questões }\end{array}$ & $\begin{array}{c}\text { Pré-teste (todos } \\
\text { participantes) }\end{array}$ & $\begin{array}{c}\text { Pós-teste grupo } \\
\text { intervenção }(\mathrm{n})\end{array}$ & $\begin{array}{c}\text { Pós-teste grupo } \\
\text { controle }(\mathrm{n})\end{array}$ \\
\hline 6 & 2 & 0 & 0 \\
7 & 3 & 2 & 2 \\
8 & 9 & 2 & 2 \\
9 & 4 & 2 & 4 \\
10 & 5 & 5 & 4 \\
\hline
\end{tabular}

Fonte: Dados da pesquisa, 2017.

Tabela 2. Média de acertos no pré-teste e no pós-teste entre os grupos controle e intervenção

\begin{tabular}{lccc}
\hline \multicolumn{1}{c}{ Grupo } & Pré-teste & Pós-teste & P-valor \\
\hline Média de acertos todos participantes & 8,30 & 8,87 & $0,029^{*}$ \\
Média de acertos grupo intervenção & 8,63 & 8,91 & 0,391 \\
Média de acertos grupo controle & 8,00 & 8,83 & $0,044^{*}$ \\
\hline
\end{tabular}

Legenda: *significativo para o teste t pareado.

Fonte: Dados da pesquisa, 2017.

\section{DISCUSSÃO}

Nessa perspectiva, os resultados desse estudo revelam que o conhecimento cognitivo em relação à assistência de enfermagem no cuidado paliativo para o grupo controle e intervenção se modificaram quando comparados ao primeiro momento, porém o grupo que vivenciou a simulação apresentou maior escore apesar de não ter sido significativo quando comparado ao grupo controle, sugerindo que a técnica pôde ter auxiliado no ensino sobre a assistência paliativa. 
Similarmente a atual pesquisa, o estudo de outros autores(11), que fizeram a utilização da mesma ferramenta para o ensino de cuidado paliativo na enfermagem, evidenciou que o grupo submetido à simulação com um paciente padronizado adquiriu maior conhecimento sobre habilidades e cuidados paliativos quando comparados ao grupo controle, o qual não participou da atividade simulada(11). Nossos resultados são consistentes com o estudo citado aventando que a estratégia de ensino adotada no grupo intervenção possa ser vislumbrada como uma possibilidade a ser adotada no ensino da graduação de enfermagem e que associá-la a outros métodos de aprendizagem pode ser promissor para 0 desenvolvimento das competências esperadas de um egresso.

Atividades simuladas parecem ser aplicáveis e capazes de favorecer o ensino sobre cuidado paliativo aos estudantes de graduação em enfermagem(12,13), porém outras estratégias de docência também são importantes neste processo, uma vez que, pelo estudo atual, os discentes que foram contemplados pelo método tradicional de ensino também melhoraram seu desempenho quando comparados entre os dois momentos. No ensino de adultos e na área da saúde uma única estratégia pode não ser suficiente para aquisição do conhecimento(14), o que justifica a melhora do escore dos estudantes que não participaram da simulação no pós-teste.

O método de ensino tradicional, por meio da aula expositiva, estimula a participação dos estudantes, porém com a simulação realística eles aprendem pela prática, pelo assemelhar ao real. No debriefing, o discente reflete sobre seu aprendizado(15). Além disso, a simulação permite ao graduando aproximar-se da realidade, do paciente e da família, mesmo que esses sejam pacientes simulados(12).

Por meio da simulação é possível ao estudante observar seu desempenho, aprender com fragilidades e fortalezas, compreender processos, trocar experiências e refletir sobre seu aprendizado oportunizando a formação crítica reflexiva desejada e esperada do egresso(2,15). Tratando-se de cuidados paliativos em que o aprender biológico não dará conta do atendimento integral ao paciente, essa estratégia demonstrou ser eficaz como nesta pesquisa, o que pode auxiliar no processo de formação dos graduandos de enfermagem $(11,12,15)$.

Por ser sujeito ativo da aprendizagem, com a simulação o estudante melhora o cumprimento de metas, fortalece o trabalho em equipe, a liderança e a tomada de decisão, desenvolve o pensamento crítico-reflexivo-ági|(15), sendo capaz de oferecer respostas rápidas diante dos desafios de assistir um paciente em tratamento paliativo. Estudos apontam que a simulação realística vem, ao longo dos anos, conseguindo um espaço no ensino e no processo de educar, melhorando assim, a formação de estudantes de enfermagem e de outros cursos na área da saúde. Relacionado aos cuidados paliativos, um outro estudo aponta que a simulação, se usada de forma organizada, pode favorecer 0 alcance das habilidades no atendimento ao paciente portador de câncer(16-19). 
Participar de diferentes estratégias de ensino propiciou aos estudantes adquirirem conhecimento cognitivo sobre a assistência de enfermagem em cuidados paliativos como notado nos escores ao comparar ambos os grupos pré e pós-testes. $O$ diferencial neste estudo ficou por conta do que pode ser alcançado com a estratégia da simulação como evidenciado em outras pesquisas(20-22). A comunicação entre equipe, entre familiares e com o paciente em tratamento paliativo será mais bem alcançada pela estratégia da simulação, pois ela propicia a interação com o paciente simulado e a possibilidade de realizar cuidados que fogem à execução de habilidades psicomotoras(11).

Durante o debriefing os estudantes participaram ativamente no processo, discutindo e refletindo sobre 0 atendimento realizado pelos mesmos, no que se refere às medidas para melhorar a qualidade de vida de um paciente em tratamento paliativo. $O$ debriefing na simulação deve propiciar um momento de aprendizado para o estudante, sendo essa a fase em que ele irá refletir sobre seu atendimento(15,23).

Compreende-se que, para cuidar de pacientes sem possibilidade de cura é necessário que a abordagem desse conteúdo seja inserida na graduação e que os estudantes de enfermagem possam vivenciar cenários semelhantes a situações reais, com as quais será possível se aproximar verdadeiramente de determinados conteúdos e refletir sobre 0 aprendizado(11,24). Esses fatores podem ser determinantes para maior aprendizado do estudante para atuar no cuidado aos pacientes em tratamento paliativo.

\section{Limitações do estudo}

Como limitações do estudo destacam-se as relacionadas à pesquisa ser realizada em uma única instituição de ensino e o tamanho da amostra, os quais podem não ter sido suficientes para identificar uma diferença significante de desempenho entre os grupos de estudantes e, além disso, ter grupos de estudantes de períodos distintos pode ter sido outro fator que interferiu no desempenho deles, uma vez que os estudantes do sexto e sétimo períodos já haviam cursado a disciplina de Enfermagem Oncológica e os demais, do quarto e quinto períodos, ainda não a haviam cursado.

A ausência de significância estatística no desempenho dos estudantes quando comparados seus escores no pós-teste pode ter sido resultante do instrumento aplicado e do momento da coleta de dados, sugerindo-se que se utilize a avaliação de habilidades clínicas estruturadas em pesquisas futuras sobre o tema quando o ensino se fizer por meio da simulação e que os dados sejam coletados a médio e longo prazo. 


\section{Contribuições para a área da enfermagem}

Por se tratar de uma simulação de baixa complexidade e com alto grau de subjetividade, fatores individuais podem interferir nos resultados, entretanto, esta pesquisa pode contribuir para a adoção de novas ferramentas de ensino na enfermagem, destinadas não somente à assistência de enfermagem paliativa, mas às formações generalistas dos enfermeiros. Ressalta-se que para a adoção desse método é preciso que docentes e instituições formadoras se envolvam com este processo, ou seja, é necessário romper com paradigmas tradicionais de ensino para propiciar melhor formação na enfermagem.

\section{CONCLUSÃO}

Os resultados desse estudo sugerem que a estratégia da simulação realística foi efetiva no ensino de cuidados paliativos na enfermagem, uma vez que ficou evidenciado no grupo intervenção um escore positivo entre um primeiro e o segundo momento quando avaliados por meio de um teste cognitivo.

Relacionada à sessão de simulação foi visível a satisfação dos estudantes com essa ferramenta no processo de ensino, indicando a necessidade de seguir rigorosamente todas as etapas do método para que o aprendizado seja reflexivo e capaz de promover mudanças.

Enfim, a inclusão da simulação realística para o ensino de cuidados paliativos na graduação em enfermagem pode resultar na formação de profissionais competentes para o cuidado com os pacientes fora de possibilidade de cura, visto que nos próximos anos se vislumbra aumento significativo desses, os quais estarão alocados nos diversos ambientes onde a assistência dos profissionais enfermeiros se fizer presente.

\section{REFERÊNCIAS}

1. Coelho MP, Menezes HF, Rosas AMMTF, Rosa AF, Pinto ACS, Saraiva RJ. Teaching in nursing care of undergraduate courses in Brazil: integrative review. Rev Enferm UFPE online [Internet]. 2016 [citado $2020 \quad$ Ago 8]; 10(2): 647-56. Available in: https://periodicos.ufpe.br/revistas/revistaenfermagem/article/view/11001/12362.

2. Fonseca LMM, Monteiro JCS, Aredes NDA, Bueno JV, Domingues AN, Coutinho VRD, Baptista RCN. Interdisciplinary simulation scenario in nursing education: humanized childbirth and birth. Rev Latino-Am Enfermagem [Internet]. 2020 [citado 2020 Ago 8]; 28: e3286. Available in: https://www.scielo.br/pdf/rlae/v28/0104-1169-rlae-28-e3286.pdf.

3. Conselho Nacional de Educação Câmara de Educação Superior. Resolução CNE/CES n 3 , de 7 de novembro de 2001. Institui Diretrizes Curriculares Nacionais do Curso de Graduação em 
Enfermagem. Brasilia: Ministério da Educação; 2001 [Internet]. [citado 2020 Ago 8]. Disponível em: http://portal.mec.gov.br/cne/arquivos/pdf/CES03.pdf.

4. Silva FC, Cunha C, Cunha C, Rodrigues T, Feitosa G, Silva AD, Sousa ID. Assistência de enfermagem a pacientes com câncer em cuidados paliativos: revisão integrativa. Revista Enfermagem Atual In Derme [Internet]. 2020 [citado 2020 Ago 8]; 91(29): 11. Disponível em: https://revistaenfermagematual.com.br/index.php/revista/article/view/626.

5. Sapeta P. Formação em cuidados paliativos: uma determinante no futuro dos cuidados de saúde. Cuidados Paliativos [Internet]. 2016 [citado 2020 Ago 8]; 3(2): 5-6. Disponível em: http://educacao.heufpel.com.br/wpcontent/uploads/sites/2/2017/08/Revista_Cuidados_Paliativos_V3_N2_web.pdf

6. Andreotti ET, Garin NC, Timm EZ. A abordagem do projeto pedagógico do curso de Enfermagem de uma instituição de ensino superior sobre o enfrentamento da morte e do morrer, na percepção dos docentes. Ciência em Movimento [Internet]. 2015 [citado 2020 Ago 8]; 17(34): 9-19. Disponível em: https://www.metodista.br/revistas/revistas-ipa/index.php/EDH/article/download/308/238.

7. World Health Organization. WHO Definition of Palliative Care [Internet]. [citado 2020 Ago 08]. Available in: http://www.who.int/cancer/palliative/definition/en/.

8. Teixeira CRS, Pereira MCA, Kusumotal L, Gaiosoll VP, Mello CL, Carvalho EC. Evaluation of nursing students about learning with clinical simulation. Rev Bras Enf [Internet]. 2015; [citado 2020 Ago 8]; 68(2): 311-9. Available in: https://www.scielo.br/pdf/reben/v68n2/en_0034-7167-reben-6802-0311.pdf.

9. Lippe MP, Becker H. Improving Attitudes and Perceived Competence in Caring for Dying Patients: An End-of-Life Simulation. Nurs Educ Perspect. 2015 Nov-Dec; [citado 2020 Oct 20]; 36(6): 372-8. Available in: http://web-a ebscohost.ez25.periodicos.capes.gov.br/ehost/pdfviewer/pdfviewer?vid=3\&sid=d22fdf76-726b4991-932a-61bb48966191\%40sessionmgr4006.

10. Pineli PP, Krasilci S, Suzuki FA, Maciel MGS. Cuidado paliativo e diretrizes curriculares: inclusão necessária. Rev Bras Educ Med [Internet]. 2017 [citado 2020 Ago 8]; 40(4): 540-6. Disponível em: https://www.scielo.br/pdf/rbem/v40n4/1981-5271-rbem-40-4-0540.pdf.

11. Tamaki T, Inumaru A, Yokoi Y, et al. The effectiveness of end-of-life care simulation in undergraduate nursing education: a randomized controlled trial. Nurse Educ Today. 2019 [citado 2020 Ago 8]; 76 : 1-7. Available in: https://www.sciencedirect.com/science/article/pii/S0260691718303381?via\%3Dihub. 
12. Lewis C, Reid J, McLernon Z, et al. The impact of a simulated intervention on attitudes of undergraduate nursing and medical students towards end of life care provision. BMC Palliat Care. 2016 [citado 2020 Oct 20]; 15(67): 1-6. 76:1-7. Available in: https://bmcpalliatcare.biomedcentral.com/articles/10.1186/s12904-016-0143-2

13. Kirkpatrick AJ, Cantrell MA, Smeltzer SC. Relationships among nursing student palliative care knowledge, experience, self-awareness, and performance: An end-of-life simulation study. Nurse Educ Today. 2019 [citado 2020 Oct 20]; 73: 23-30. Available in: https://wwwsciencedirect.ez25.periodicos.capes.gov.br/search?qs=Relationships $\% 20$ among\%20nursing\%20st udent $\% 20$ palliative $\% 20$ care $\% 20$ knowledge $\% 2 \mathrm{C} \% 20$ experience $\% 2 \mathrm{C} \% 20$ self-

awareness $\% 2 \mathrm{C} \% 20$ and $\% 20$ performance $\% 3 \mathrm{~A} \% 20 \mathrm{An} \% 20$ end-of life $\% 20$ simulation $\% 20$ study\&pub=Nurse $\% 20$ Education $\% 20$ Today \&cid=272471

14. Pedersoli CE, Martins-Pedersoli TA, Faro ACM, Dalri MCB. Teaching airway management with laryngeal mask: randomized controlled trial. Rev Bras Enferm [Internet]. 2016 [citado 2020 Ago 8]; 69(2): 345-51. Available in: https://www.scielo.br/pdf/reben/v69n2/en_0034-7167-reben-69-020368.pdf.

15. Gaba DM. The future vision of simulation in health care. Qual Saf Health Care [Internet]. 2004 [citado 2020 Ago 8]; 13(Suppl 1): 2-10. Available in: https://qualitysafety.bmj.com/content/qhc/13/suppl_1/i2.full.pdf.

16. Lavoie $P$, Michaud $C$, Bélisle $M$, et al. Learning theories and tools for the assessment of core nursing competencies in simulation: a theoretical review. J Adv Nurs [Internet]. 2018 [citado 2020 Ago 8]; 74(2): 239-250. Available in: https://onlinelibrary.wiley.com/doi/epdf/10.1111/jan.13416.

17. Bortolato-Major C, Arhur JP, Mattei AT, Mantovani MF, Felix JVC, Boostel R. Contributions of the simulation for undergraduate nursing students. Rev Enferm UFPE On Line [Internet]. 2018 [citado 2020 Ago 8]; 12(6): 1751-62. Available in: https://periodicos.ufpe.br/revistas/revistaenfermagem/article/view/230633/29224.

18. Guimarães TM, Silva LF, Santo FHE, Moraes JRMM. Palliative care in pediatric oncology in nursing students' perception. Esc Anna Nery [Internet]. 2016 [citado 2020 Ago 8]; 20(2): 261-7. Available in: https://www.scielo.br/pdf/ean/v20n2/en_1414-8145-ean-20-02-0261.pdf.

19. Valadares AF, Magro MC. Opinion of nursing students on realistic simulation and the curriculum internship in hospital setting. Acta Paul Enferm [Internet]. 2014 [citado 2020 Ago 8]; 27(2): 138-43. Available in: https://www.scielo.br/pdf/ape/v27n2/en_0103-2100-ape-27-02-0138.pdf.

20. Akhu-Zahega LM, Gharaibeh MK, Alostaz ZM. Effectiveness of simulation on knowledge acquisition, knowledge retention, and self-efficacy of nursing students in Jordan. Clin Simul Nurs [Internet]. 2013 
[citado 2020 Ago 8]; 9(9): 335-42. Available in: https://www.sciencedirect.com/science/article/abs/pii/S1876139912000710.

21. Sarabia-Cobo CM, Alconero-Camarero AR, Lavín-Alconero L, Ibáñez-Remetería I. Assessment of a learning intervention in palliative care based on clinical simulations for nursing students. Nurse Educ Today [Internet]. 2016 [citado 2020 Ago 8]; 45: 219-24. Available in: https://www.sciencedirect.com/science/article/abs/pii/S0260691716301617?via\%3Dihub.

22. Shaw PA, Abbott MA. High-fidelity simulation: teaching end-of-life care. Nurse Educ Today [Internet]. 2017 [citado 2020 Ago 8]; 49: 8-11. Available in: https://www.sciencedirect.com/science/article/abs/pii/S0260691716302520?via\%3Dihub.

23. Maestre JM, Rudolph JW. Theories and styles of debriefing: the good judgment method as a tool for formative assessment in healthcare. Rev Esp Cardiol [Internet]. 2015 [citado 2020 Ago 8]; 68(4): 282-5. Available in: https://www.revespcardiol.org/en-pdf-S1885585714002989.

24. Rodriguez KG, Nelson N, Gilmartin M, Goldsamt L, Richardson H. Simulation is more than working with a mannequin: student's perceptions of their learning experience in a clinical simulation environment. J Nurs Educ Pract [Internet]. 2017 [citado 2020 Ago 8]; 7(7): 30-6. Available in: http://www.sciedupress.com/journal/index.php/jnep/article/download/10232/6758. 